\title{
ASYMPTOTIC BEHAVIOR AND STRONG CONVERGENCE FOR HYPERBOLIC SYSTEMS OF CONSERVATION LAWS WITH DAMPING
}

\author{
BY \\ CORRADO LATTANZIO AND BRUNO RUBINO
}

Dipartimento di Matematica Pura ed Applicata, Università degli Studi di L'Aquila, via Vetoio, loc. Coppito - 67010 L'Aquila, Italy

\begin{abstract}
A local type estimate will be proved here for general $2 \times 2$ hyperbolic systems of conservation laws with strong dissipative term. Following the idea of [15], this result will be achieved by using as a preliminary step the convergence in the mean, which is an immediate consequence of the result of [11] obtained by using the compensated compactness theory.
\end{abstract}

1. Introduction. In this paper we investigate the asymptotic behavior of solutions for the nonlinear system of partial differential equations

$$
\left\{\begin{array}{l}
w_{s}+f(w, z)_{y}=0 \\
z_{s}+g(w, z)_{y}=h(w, z),
\end{array}\right.
$$

and we prove that, under the usual parabolic rescaling,

$$
\left\{\begin{array}{l}
u^{\varepsilon}(x, t)=w^{\varepsilon}\left(\frac{x}{\sqrt{\varepsilon}}, \frac{t}{\varepsilon}\right) \\
v^{\varepsilon}(x, t)=\frac{1}{\sqrt{\varepsilon}} z^{\varepsilon}\left(\frac{x}{\sqrt{\varepsilon}}, \frac{t}{\varepsilon}\right),
\end{array}\right.
$$

passing into the limit $\varepsilon \downarrow 0$, this system is described by a porous media type equation

$$
u_{t}-\beta(u)_{x x}=0, \quad \beta^{\prime}(u)=-f^{*}(u) h^{*}(u)^{-1}\left(g^{0}(u)\right)^{\prime},
$$

where $f^{*}(w)=\frac{\partial f}{\partial z}(w, 0), h^{*}(w)=\frac{\partial h}{\partial z}(w, 0)$, and $g^{0}(w)=g(w, 0)$.

Received February 15, 2003.

2000 Mathematics Subject Classification. Primary 35L65; Secondary 35L40, 76R50.

Key words and phrases. compensated compactness, conservation law, energy estimate, entropy inequality, hyperbolic system, nonlinear dissipative equation, porous media equation, relaxation, rescaling. Partially supported by European Union - RTN Grant HPRN-CT-2002-00282 (HYKE European Network) and MIUR-COFIN-2002, Progetto Nazionale "Equazioni Iperboliche e Paraboliche Nonlineari".

E-mail address: corrado@univaq.it

E-mail address: rubino@univaq.it 
We will consider initial data in $L^{\infty}$ and, by using compensated compactness first and then energy estimates, we will prove strong convergence in $L_{l o c}^{2}$, as the time tends to infinity, for large weak entropy solutions of (1) towards his profile, which is the unique similarity solution of (3). We postpone the precise statement and assumptions to Sec. 2 .

The problem of relaxation from hyperbolic to parabolic systems is relevant in many situations in applied mathematics. A way to understand the meaning of these phenomena is to consider it as the large time behavior of dissipative nonlinear hyperbolic systems and to look at the asymptotic profile as the relaxed equilibrium.

There is extensive literature concerning this kind of investigation but the techniques are different when we refer to the case of regular or weak solutions. Among these, we want to mention that, by using energy estimates, Hsiao and T.-P. Liu [2] studied convergence to nonlinear diffusion waves for solutions of a system of hyperbolic conservation laws with damping, in the case of small and smooth initial data. If we refer to the case of weak solutions, a large number of results have been obtained by using the techniques of compensated compactness. Here we want to mention the papers of Marcati and Milani [8] for the case of the relaxation of the $2 \times 2$ isentropic, damped, compressible Euler equations to the equations describing the porous media flow; Marcati, Milani, and Secchi [9] for the relaxation of the $2 \times 2 p$-system model with damping to nonlinear heat conduction; and Lions and Toscani [7], where the hydrodynamic version of the Carleman equations has been shown to converge towards a nonlinear diffusion equation, which plays the role of the natural hydrodynamic limit. Finally, this is also the case of Marcati and Rubino [11] where the interest is to study, in a systematic way, these kinds of hyperbolic to parabolic relaxation limits for general $2 \times 2$ strong dissipative systems.

Starting from the relaxation result obtained in [9], Hsiao and D. Serre in [15] proved a stronger convergence result for solutions, which they called strong convergence in $L_{\text {loc }}^{2}$ as a refinement of the $L_{l o c}^{p}$ convergence in the mean obtained in [9]. Similarly, Huang and Pan in [4] proved this kind of stronger convergence in connection with the result of $[8]$.

The idea in this paper is to obtain results similar to [15] and [4] for the general $2 \times 2$ strictly hyperbolic systems in $1-D$ by coupling the convergence obtained in [11] by means of compensated compactness arguments with energy estimates. Although in [11] other cases are also treated, in this paper these limitations are essential because of the difficulties concerning the properties of the similarity solution for the limiting equations.

Section 2 of this paper is dedicated to reformulating the problem for the general strong dissipative system (1) and to recall the results of [11] we need here. Then in Sec. 3 we will obtain the energy type estimates and we will prove the strong convergence result.

2. Formulation of the problem and the mean convergence. In this section we will recall some results established in [11] concerning the existence of the weak entropy solutions of (1) and the limit, as $\varepsilon \downarrow 0$, of these solutions toward the solution of the parabolic equation (3). For this purpose, let us consider the following system of balance 
laws (1):

$$
\left\{\begin{array}{l}
w_{s}+f(w, z)_{y}=0 \\
z_{s}+g(w, z)_{y}=h(w, z)
\end{array}\right.
$$

with initial data $w_{0}, z_{0} \in L^{\infty}(\mathbb{R})$. For the system (1), we assume the following hypotheses (see [11]) hold:

(S.1) $f, g, h: \mathbb{R}^{2} \longrightarrow \mathbb{R}$ are $C^{1}$ functions such that

$$
f(w, 0)=h(w, 0)=0 ;
$$

(S.2) the system is strictly hyperbolic, namely

$$
\left(f_{w}+g_{z}\right)^{2}+4 g_{w} f_{z}>0
$$

(S.3) if we denote by $f^{*}(w)=\left.\frac{\partial f}{\partial z}\right|_{z=0}, h^{*}(w)=\left.\frac{\partial h}{\partial z}\right|_{z=0}$ and $g^{0}(w)=g(w, 0)$, then

$$
h^{*}(w)<0, \quad\left(g^{0}(w)\right)^{\prime} f^{*}(w)>0 ;
$$

(S.4) there exist a $C^{2}$ strictly convex entropy-entropy flux pair $\left(\eta^{*}, q^{*}\right)$, a constant $\gamma>0$, and a $C^{2}$ function $\psi$ satisfying $\psi^{-1}(\{0\})=\{0\}, \psi^{\prime \prime}>2 \gamma$ such that

$$
\eta_{z}^{*}(w, z) h(w, z) \leq-\psi(z) .
$$

REMARK 1. Note that condition (S.4) implies, by standard calculus arguments, $\psi^{\prime}(0)=0$ because $\psi$ is a uniform strictly convex function which vanishes only for $z=0$. Therefore $\psi$ verifies $\psi(z)>\gamma z^{2}$ for any $z \in \mathbb{R} \backslash\{0\}$.

REMARK 2. The structural conditions (S.1) through (S.4) are intended to give a framework of models where this kind of analysis can be carried out. Clearly, the damped $p$-system studied in [15] enters in this framework and it is in fact the starting point of this generalization. Moreover, the simplest models which fulfill our conditions can be obtained, for instance, as uncoupled perturbations of the damped $p$-system of the form

$$
\left\{\begin{array}{l}
w_{s}+f(z)_{y}=0 \\
z_{s}+g(w)_{y}=h(z)
\end{array}\right.
$$

with

$$
\begin{aligned}
& f(z)=z+f_{1}(z) \\
& h(z)=-z-h_{1}(z)
\end{aligned}
$$

with $g^{\prime}(w)>0, f_{1}(0)=h_{1}(0)=0$ and $z f_{1}(z) \geq 0, z h_{1}(z) \geq 0$ for any $z \neq 0$. Hence, a strictly convex entropy $\eta^{*}(w, z)$ is explicitly given by

$$
\eta^{*}(w, z)=\frac{1}{2} z^{2}+\int^{z} f_{1}(\zeta) d \zeta+\int^{w} g(\xi) d \xi
$$

and condition (S.4) is fulfilled with $\psi(z)=z^{2}$.

Under the parabolic rescaling (2), the system (1) rewrites

$$
\left\{\begin{array}{l}
u_{t}^{\varepsilon}+\frac{1}{\sqrt{\varepsilon}} f\left(u^{\varepsilon}, \sqrt{\varepsilon} v^{\varepsilon}\right)_{x}=0 \\
\varepsilon v_{t}^{\varepsilon}+g\left(u^{\varepsilon}, \sqrt{\varepsilon} v^{\varepsilon}\right)_{x}=\frac{1}{\sqrt{\varepsilon}} h\left(u^{\varepsilon}, \sqrt{\varepsilon} v^{\varepsilon}\right),
\end{array}\right.
$$


while the initial data becomes

$$
\left\{\begin{array}{l}
u_{0}^{\varepsilon}(x)=w_{0}\left(\frac{x}{\sqrt{\varepsilon}}\right) \\
v_{0}^{\varepsilon}(x)=\frac{1}{\sqrt{\varepsilon}} z_{0}\left(\frac{x}{\sqrt{\varepsilon}}\right) .
\end{array}\right.
$$

For the rescaled initial conditions (5), we assume that the following convergence holds:

(S.5) $u_{0}^{\varepsilon} \stackrel{*}{\rightarrow} \bar{u}$ in $L^{\infty}$ when $\varepsilon \downarrow 0$, where

$$
\bar{u}= \begin{cases}w_{l} & \text { if } x<0 \\ w_{r} & \text { if } x>0\end{cases}
$$

Finally, concerning the Cauchy problem associated with (1), we assume

(S.6) there exists a weak entropy solution to the Cauchy problem for (1), obtained with the vanishing viscosity method, bounded in $L^{\infty}\left(\mathbb{R} \times \mathbb{R}^{+}\right)$.

The scaling (2) transforms the solution to the Cauchy problem for (1) given in (S.6) into a weak entropy solution $\left(u^{\varepsilon}, v^{\varepsilon}\right)$ of the rescaled system (4), verifying

$$
\left\|u^{\varepsilon}\right\|_{\infty}, \sqrt{\varepsilon}\left\|v^{\varepsilon}\right\|_{\infty} \leq C .
$$

More precisely, the pair $\left(u^{\varepsilon}, v^{\varepsilon}\right)$ verifies the following relations:

$$
\begin{aligned}
& \iint_{t>0}\left(u^{\varepsilon} \Phi_{t}+\frac{1}{\sqrt{\varepsilon}} f\left(u^{\varepsilon}, \sqrt{\varepsilon} v^{\varepsilon}\right) \Phi_{x}\right) d x d t+\int_{t=0} u_{0}^{\varepsilon} \Phi(x, 0) d x=0, \\
& \iint_{t>0}\left(\varepsilon v^{\varepsilon} \Phi_{t}+g\left(u^{\varepsilon}, \sqrt{\varepsilon} v^{\varepsilon}\right) \Phi_{x}-\frac{1}{\sqrt{\varepsilon}} h\left(u^{\varepsilon}, \sqrt{\varepsilon} v^{\varepsilon}\right) \Phi\right) d x d t \\
& +\int_{t=0} \varepsilon v_{0}^{\varepsilon} \Phi(x, 0) d x=0,
\end{aligned}
$$

for any test function $\Phi$ with compact support, intersecting the axis $t=0$. Hence, the pair $\left(u^{\varepsilon}, v^{\varepsilon}\right)$ is a weak solution of (4) with $\left(u_{0}^{\varepsilon}, \varepsilon v_{0}^{\varepsilon}\right)$ as initial condition. We procede now with the study of the entropy inequality that the weak solution of (4) must verify. Let $(E(w, z), Q(w, z))$ be a convex entropy-entropy flux pair for (1), namely

$$
\begin{aligned}
& \iint_{s>0}\left(E(w, z) \Phi_{s}+Q(w, z) \Phi_{y}-E_{z}(w, z) h(w, z) \Phi\right) d y d s \\
& +\int_{s=0} E(y, 0) \Phi(y, 0) d y \geq 0,
\end{aligned}
$$

for any test function $\Phi \geq 0$ with compact support intersecting the axis $t=0$. As it was pointed out in [11], a corresponding entropy-entropy flux pair $\left(\eta^{\varepsilon}\left(u^{\varepsilon}, v^{\varepsilon}\right), q^{\varepsilon}\left(u^{\varepsilon}, v^{\varepsilon}\right)\right)$ for (4) is given by

$$
\begin{aligned}
& \eta^{\varepsilon}\left(u^{\varepsilon}, v^{\varepsilon}\right)=\sqrt{\varepsilon} E\left(u^{\varepsilon}, \sqrt{\varepsilon} v^{\varepsilon}\right) \\
& q^{\varepsilon}\left(u^{\varepsilon}, v^{\varepsilon}\right)=Q\left(u^{\varepsilon}, \sqrt{\varepsilon} v^{\varepsilon}\right) .
\end{aligned}
$$


Moreover, the entropy inequality becomes

$$
\begin{aligned}
& \iint_{t>0}\left(\eta^{\varepsilon}\left(u^{\varepsilon}, v^{\varepsilon}\right) \Phi_{t}+q^{\varepsilon}\left(u^{\varepsilon}, v^{\varepsilon}\right) \Phi_{x}\right. \\
& \left.-\frac{1}{\varepsilon \sqrt{\varepsilon}} \eta_{v}^{\varepsilon}\left(u^{\varepsilon}, v^{\varepsilon}\right) h\left(u^{\varepsilon}, \sqrt{\varepsilon} v^{\varepsilon}\right) \Phi\right) d x d t+\int_{t=0} \eta^{\varepsilon}(x, 0) \Phi(x, 0) d x \geq 0 .
\end{aligned}
$$

By rewriting the above relation in terms of the original entropy-entropy flux pair $(E, Q)$, we get

$$
\begin{aligned}
& \iint_{t>0}\left(\sqrt{\varepsilon} E\left(u^{\varepsilon}, \sqrt{\varepsilon} v^{\varepsilon}\right) \Phi_{t}+Q\left(u^{\varepsilon}, \sqrt{\varepsilon} v^{\varepsilon}\right) \Phi_{x}\right. \\
& \left.-\frac{1}{\sqrt{\varepsilon}} E_{z}\left(u^{\varepsilon}, \sqrt{\varepsilon} v^{\varepsilon}\right) h\left(u^{\varepsilon}, \sqrt{\varepsilon} v^{\varepsilon}\right) \Phi\right) d x d t+\int_{t=0} \sqrt{\varepsilon} E(x, 0) \Phi(x, 0) d x \geq 0,
\end{aligned}
$$

for any convex entropy-entropy flux pair $(E(w, z), Q(w, z))$ of $(1)$ and for any test function $\Phi \geq 0$ with compact support intersecting the axis $t=0$.

We start by collecting some properties of the function $\eta^{*}(w, z)$, which will be useful in proving the basic energy-type estimate of the next section.

Lemma 1. Let $a, b \in \mathbb{R}$ be two arbitrary fixed constants and let the conditions (S.3) and (S.4) hold. Then

$$
\eta_{z}^{*}(w, 0)=0, \text { for any } w \in[a, b] .
$$

In particular, there exists a function $G(w, z) \in C_{w}^{1} \cap C_{z}^{0}$ such that

$$
\eta_{z}^{*}(w, z)=z G(w, z) \text {. }
$$

Proof. By contradiction, let us assume that there exists a $w_{0} \in[a, b]$ such that $\eta_{z}^{*}\left(w_{0}, 0\right) \neq 0$. Without loss of generality, we can assume $\eta_{z}^{*}\left(w_{0}, 0\right)>0$. From (S.4) it follows

$$
\eta_{z}^{*}(w, z) h(w, z) \leq-\gamma z^{2}
$$

Since (S.3) implies

$$
\frac{\partial h}{\partial z}(w, 0)=h^{*}(w)<-k<0, \text { for any } w \in[a, b],
$$

there exist $\delta>0$ and constants $C_{1}, C_{2}>0$ such that

$$
C_{1}<-\frac{h(w, z)}{z}<C_{2}, \text { for any }|z|<\delta, w \in[a, b] .
$$

Hence, (9) and (10) yield

$$
C z^{2} \leq z \eta_{z}^{*}\left(w_{0}, z\right)=z \eta_{z}^{*}\left(w_{0}, 0\right)+z^{2} \eta_{z z}^{*}\left(w_{0}, \widetilde{z}\right),
$$

for any $|\widetilde{z}|<|z|<\delta$. Thus, with the notation $M=\sup _{|\zeta|<\delta}\left|\eta_{z z}^{*}\left(w_{0}, \zeta\right)\right|$, the inequality (11) implies

$$
a z \geq(C-M) z^{2}, \quad a=\eta_{z}^{*}\left(w_{0}, 0\right)>0,|z|<\delta,
$$

which yields a contradiction for $z$ negative and $|z|$ sufficiently small. Therefore

$$
\eta_{z}^{*}(w, 0)=0
$$


for any $w \in[a, b]$ and

$$
\eta_{z}^{*}(w, z)=z \eta_{z z}^{*}(w, \widetilde{z})=z G(w, z)
$$

for any $z$ and for any $w \in[a, b]$. Finally, the regularity of $G(w, z)=\frac{\eta_{z}^{*}(w, z)}{z}$ follows from the regularity of $\eta^{*}(w, z)$ and the proof is complete.

We conclude this section with the first convergence result for the solutions of the rescaled system (4). Thanks to the conditions (S.1), (S.2), (S.3), and (S.5), and in view of the uniform $L^{\infty}$ bound for $\sqrt{\varepsilon} v^{\varepsilon}$, passing into the limit $\varepsilon \downarrow 0$ in (7) and (8), we formally obtain

$$
\left\{\begin{array}{l}
u_{t}+\left(f^{*}(u) v\right)_{x}=0 \\
g^{0}(u)_{x}=h^{*}(u) v
\end{array}\right.
$$

which can be rewritten as the scalar parabolic equation (3)

$$
u_{t}-\beta(u)_{x x}=0, \quad \beta^{\prime}(u)=-f^{*}(u) h^{*}(u)^{-1}\left(g^{0}(u)\right)^{\prime}>0,
$$

with initial condition

$$
u(x, 0)=\bar{u}(x)= \begin{cases}w_{l} & \text { if } x<0 \\ w_{r} & \text { if } x>0 .\end{cases}
$$

In order to prove the strong convergence of $u^{\varepsilon}$ toward its formal limit, we need an $L^{2}$ control of $v^{\varepsilon}$. Due to our condition (S.5) on the initial data, we can not apply directly the result of [11], because, in this case, the finite energy condition

$$
\int_{-\infty}^{+\infty} \eta^{*}\left(u_{0}^{\varepsilon}, \sqrt{\varepsilon} v_{0}^{\varepsilon}\right) d x<c
$$

cannot be fulfilled. However, even without this condition, it is still possible to get an $L_{l o c}^{2}$ estimate for $v^{\varepsilon}$, which is enough to apply the compensated compactness framework of [11] and thus to obtain the strong convergence.

Lemma 2. Let us assume that the hypotheses (S.1) through (S.6) hold and let $\left(u^{\varepsilon}, v^{\varepsilon}\right)$ be a weak entropy solution of (4) with $\left(u_{0}^{\varepsilon}, \varepsilon v_{0}^{\varepsilon}\right)$ as initial datum. Then

$$
\int_{0}^{T} \int_{-L}^{+L}\left|v^{\varepsilon}\right|^{2} d x d t<C,
$$

where the positive constant $C$ is independent from $\varepsilon$.

Proof. The entropy inequality for the pair $\left(\eta^{*}, q^{*}\right)$ yields to the following relation:

$$
\begin{aligned}
& \iint_{t>0}\left(\eta^{*}\left(u^{\varepsilon}, \sqrt{\varepsilon} v^{\varepsilon}\right) \Phi_{t}+\frac{1}{\sqrt{\varepsilon}} q^{*}\left(u^{\varepsilon}, \sqrt{\varepsilon} v^{\varepsilon}\right) \Phi_{x}\right. \\
& \left.-\frac{1}{\varepsilon} \eta_{z}^{*}\left(u^{\varepsilon}, \sqrt{\varepsilon} v^{\varepsilon}\right) h\left(u^{\varepsilon}, \sqrt{\varepsilon} v^{\varepsilon}\right) \Phi\right) d x d t+\int_{t=0} \eta^{*}(x, 0) \Phi(x, 0) d x \geq 0,
\end{aligned}
$$

where $\Phi \geq 0$ is any test function with compact support intersecting the axis $t=0$. The condition (S.4) implies

$$
\eta_{z}^{*}\left(u^{\varepsilon}, \sqrt{\varepsilon} v^{\varepsilon}\right) h\left(u^{\varepsilon}, \sqrt{\varepsilon} v^{\varepsilon}\right) \leq-\psi\left(\sqrt{\varepsilon} v^{\varepsilon}\right) \leq-\gamma \varepsilon\left|v^{\varepsilon}\right|^{2}
$$


and therefore (14) becomes

$$
\begin{aligned}
\gamma \iint_{t>0}\left|v^{\varepsilon}\right|^{2} \Phi d x d t & \leq \iint_{t>0} \eta^{*}\left(u^{\varepsilon}, \sqrt{\varepsilon} v^{\varepsilon}\right) \Phi_{t} d x d t \\
+\int_{t=0} \eta^{*}(x, 0) \Phi(x, 0) d x & +\iint_{t>0} \frac{1}{\sqrt{\varepsilon}} q^{*}\left(u^{\varepsilon}, \sqrt{\varepsilon} v^{\varepsilon}\right) \Phi_{x} d x d t \\
& \leq C+\iint_{t>0} \frac{1}{\sqrt{\varepsilon}} q^{*}\left(u^{\varepsilon}, \sqrt{\varepsilon} v^{\varepsilon}\right) \Phi_{x} d x d t
\end{aligned}
$$

in view of the uniform $L^{\infty}$ bound for $u^{\varepsilon}$ and for $\sqrt{\varepsilon} v^{\varepsilon}$. Since condition (S.1) and Lemma 1 imply $q_{w}(w, 0)=0$ for any entropy flux $q$ of (1), without loss of generality, we can assume, $q^{*}(w, 0)=0$. Thus,

$$
\frac{1}{\sqrt{\varepsilon}}\left|q^{*}\left(u^{\varepsilon}, \sqrt{\varepsilon} v^{\varepsilon}\right)\right|=\frac{1}{\sqrt{\varepsilon}}\left|q_{w}^{*}\left(u^{\varepsilon}, \zeta\right)\right|\left|\sqrt{\varepsilon} v^{\varepsilon}\right| \leq C\left|v^{\varepsilon}\right|
$$

and (15) yields to

$$
\begin{aligned}
\iint_{t>0}\left|v^{\varepsilon}\right|^{2} \Phi d x d t & \leq C+C \iint_{t>0}\left|v^{\varepsilon}\right|\left|\Phi_{x}\right| d x d t \\
& \leq C+C \iint_{t>0} \frac{\left|\Phi_{x}\right|^{2}}{\Phi} d x d t+\frac{1}{2} \iint_{t>0}\left|v^{\varepsilon}\right|^{2} \Phi d x d t
\end{aligned}
$$

Hence, we have proved the following estimate:

$$
\iint_{t>0}\left|v^{\varepsilon}\right|^{2} \Phi d x d t \leq C+C \iint_{t>0} \frac{\left|\Phi_{x}\right|^{2}}{\Phi} d x d t .
$$

Now we choose $\Phi(x, t)=\alpha(x) \beta(t)$ such that $\alpha \equiv 1$ for $x \in[-L, L], \beta \equiv 1$ for $t \in[0, T]$, and such that $\frac{\left|\alpha^{\prime}\right|^{2}}{\alpha}$ is bounded. Finally,

$$
\begin{aligned}
\int_{0}^{T} \int_{-L}^{+L}\left|v^{\varepsilon}\right|^{2} d x d t & \leq \iint_{t>0}\left|v^{\varepsilon}\right|^{2} \Phi d x d t \\
& \leq C+C \iint_{t>0} \beta(t) \frac{\left|\alpha^{\prime}\right|^{2}}{\alpha} d x d t \\
& \leq C
\end{aligned}
$$

which concludes the proof.

Now, by using arguments of [11], it is possible to prove the following convergence result.

Theorem 1. Let us assume that the hypotheses (S.1) through (S.6) hold and let $\left(u^{\varepsilon}, v^{\varepsilon}\right)$ be a weak entropy solution of $(4)$ with $\left(u_{0}^{\varepsilon}, \varepsilon v_{0}^{\varepsilon}\right)$ as initial datum. Then

$$
\begin{array}{ll}
u^{\varepsilon} \longrightarrow u & \text { strongly in } L_{l o c}^{p} \text { for any } p<+\infty \\
v^{\varepsilon} \longrightarrow v & \text { weakly in } L_{l o c}^{2} \\
\sqrt{\varepsilon} v^{\varepsilon} \longrightarrow 0 & \text { strongly in } L_{l o c}^{2},
\end{array}
$$

where $(u, v)$ verifies the system (12), the first line in $\mathcal{D}^{\prime}$, and the second in $L^{2}$. More precisely, $u(x, t)$ is the self-similar solution of the form $U\left(\frac{x}{\sqrt{1+t}}\right)$ of the Cauchy problem (3)-(13). 
REMARK 3. The results established in the theorem above can be interpreted as an asymptotic behavior for the solution of the hyperbolic system of balance laws. Indeed, let us introduce the following local error term:

$$
E(\varphi, s)=\int_{-\infty}^{+\infty} \varphi(\xi)|w(\xi \sqrt{s}, s)-U(\xi)|^{2} d \xi,
$$

where $\varphi$ is a positive test function, $\xi=\frac{y}{\sqrt{s}}$ is the self-similar variable, and $U(\xi)=u(y, s)$ is the self-similar solution of (3)-(13). Therefore, the convergence

$$
u^{\varepsilon} \longrightarrow u \text { strongly in } L_{l o c}^{p} \text { for any } p<+\infty
$$

is equivalent to the following mean convergence $([15])$ :

$$
\lim _{T \rightarrow+\infty} \frac{1}{T} \int_{0}^{T} E(\varphi, s) d s=0 .
$$

3. The energy-type estimate and the "local" convergence. The aim of this section is to improve the mean asymptotic behavior given by (17) to the following pointwise convergence:

$$
\lim _{s \rightarrow+\infty} E(\varphi, s)=0 .
$$

The main tool in performing this task will be the energy estimate used in [15] in the case of the p-system with a linear damping term. It is worth observing that in our general case the energy estimate is complicated by some terms we have to take into account, terms which are not present in the specific case discussed in [15], due to the particular structure of the p-system and due to the presence of a linear damping term.

Theorem 2. Assume that the hypotheses (S.1) through (S.6) hold and let $E(\varphi, s)$ be the local error term defined in (16). Then

$$
\lim _{s \rightarrow+\infty} E(\varphi, s)=0 .
$$

Before going into the proof of this theorem, we establish some preliminary results which we will use in this proof, following again the ideas of [15]. The next proposition gives the fundamental decay properties of the self-similar solution of the problem (3)-(13) (see $[2])$.

Proposition 1. Assume that the hypothesis (S.3) holds and let $u(x, t)=U\left(\frac{x}{\sqrt{t}}\right)$ be the self-similar solution of $(3)-(13)$. Then $U(\xi)$ is monotone and $u(x, t)$ verifies the following estimates:

$$
\begin{array}{ll}
\left|u_{t}\right| \leq \frac{C}{t} & \left|u_{x}\right| \leq \frac{C}{\sqrt{t}} \\
\left|u_{t x}\right| \leq \frac{C}{t^{\frac{3}{2}}} & \left|u_{x x}\right| \leq \frac{C}{t},
\end{array}
$$

for a given constant $C>0$. 
The next lemma gives a Gronwall-type estimate which will reduce the proof of the limit behavior (18) to the proof of a suitable energy inequality [15].

Lemma 3. Let $F: \mathbb{R}^{+} \longrightarrow \mathbb{R}^{+}$be a bounded measurable function such that

(i) $\lim _{T \rightarrow+\infty} \frac{1}{T} \int_{0}^{T} F(s) d s=0$;

(ii) there exists a constant $C \geq 0$ such that

$$
F^{\prime}(s) \leq \frac{C}{s}
$$

Then

$$
F(T) \leq C\left(\frac{1}{T} \int_{0}^{T} F(s) d s\right)^{\frac{1}{2}}
$$

In particular

$$
\lim _{s \rightarrow+\infty} F(s)=0
$$

Proof of Theorem 2. Due to Remark 3 and Lemma 3, in order to prove the relation (18), it suffices to prove the following decay estimate for the local error term given in (16):

$$
\frac{d}{d s} E(\varphi, s) \leq \frac{C}{s}
$$

for a constant $C \geq 0$ and $s$ large. To this end, let us consider the quadratic part of the entropy $\eta^{*}$ with respect to the asymptotic state $(u, v)$ :

$$
\begin{aligned}
H(y, s)= & \eta^{*}(w(y, s), z(y, s))-\eta^{*}(u(y, s), v(y, s)) \\
& -\left\langle\nabla \eta^{*}(u(y, s), v(y, s)),(w(y, s)-u(y, s), z(y, s)-v(y, s))\right\rangle .
\end{aligned}
$$

Since $\eta^{*}$ is strictly convex, the estimate (19) is proved if

$$
\frac{d}{d s} \widetilde{E}(\varphi, s) \leq \frac{C}{s}
$$

where $C \geq 0, s$ large and

$$
\widetilde{E}(\varphi, s)=\int_{-\infty}^{+\infty} \varphi(\xi) H(\xi \sqrt{s}, s) d \xi
$$

Let $Q$ be the flux associated with the entropy $H$ for the system (1), namely:

$$
\begin{aligned}
Q(y, s)= & q^{*}(w(y, s), z(y, s))-\nabla \eta^{*}(u(y, s), v(y, s)) \\
& \times(f(w(y, s), z(y, s)), g(w(y, s), z(y, s))) .
\end{aligned}
$$

Thus, the entropy inequality for the weak solutions of (1) yields

$$
\begin{aligned}
\partial_{s} H+\partial_{y} Q+I_{1} \leq & -\partial_{s}\left[\nabla \eta^{*}(u, v)\right](w-u, z-v) \\
& -\partial_{y}\left[\nabla \eta^{*}(u, v)\right](f(w, z), g(w, z)) \\
= & I_{2}+I_{3},
\end{aligned}
$$


where $I_{1}=\eta_{v}^{*}(u, v) h(w, z)-\eta_{z}^{*}(w, z) h(w, z)$. From (S.1) and (S.4) we get

$$
\begin{aligned}
I_{1} & \geq \eta_{v}^{*}(u, v) h(w, z)+\gamma z^{2} \geq-\left|\eta_{v}^{*}(u, v)\right|\left|h_{z}(w, \widetilde{z})\right||z|+\gamma z^{2} \\
& \geq-\left[C_{\gamma}\left|\eta_{v}^{*}(u, v)\right|^{2}\left|h_{z}(w, \widetilde{z})\right|^{2}+\frac{\gamma}{2} z^{2}\right]+\gamma z^{2} \\
& \geq-I_{4},
\end{aligned}
$$

where $I_{4}=C_{\gamma}\left|\eta_{v}^{*}(u, v)\right|^{2}\left|h_{z}(w, \widetilde{z})\right|^{2}$. Now, by using the $L^{\infty}$ bounds for $u, v, w$, and $z$ and by using the results of Lemma 1 and Proposition 1, one has

$$
\left|I_{2}\right| \leq C\left[\left|u_{s}\right|+\left|v_{s}\right|\right] \leq \frac{C}{s} \text { for any } s>1,
$$

since

$$
\begin{aligned}
\left|v_{s}\right| & =\left|\partial_{s}\left[h^{*}(u)^{-1} g^{0}(u)^{\prime} u_{y}\right]\right| \leq C\left(\left|u_{y s}\right|+\left|u_{y}\right|\left|u_{s}\right|\right) \\
& \leq \frac{C}{s^{\frac{3}{2}}} \leq \frac{C}{s}
\end{aligned}
$$

for any $s>1$. Moreover,

$$
\left|I_{4}\right| \leq C|v|^{2}<\frac{C}{s}
$$

because, as before,

$$
|v|^{2}=\left|h^{*}(u)^{-1}\right|^{2}\left|g^{0}(u)^{\prime}\right|^{2}\left|u_{y}\right|^{2} .
$$

At this point we need to estimate only the term

$$
I_{3}=-\eta_{u u}^{*} u_{y} f(w, z)-\eta_{u v}^{*} v_{y} f(w, z)-\eta_{u v}^{*} u_{y} g(w, z)-\eta_{v v}^{*} v_{y} g(w, z) .
$$

As before, Lemma 1 implies $\left|\eta_{u v}^{*}\right| \leq C|v|$ and therefore

$$
\left|\eta_{u v}^{*} u_{y} g(w, z)\right| \leq C|v|\left|u_{y}\right| \leq \frac{C}{s} .
$$

Moreover,

$$
\left|\eta_{u v}^{*} v_{y} f(w, z)\right|+\left|\eta_{v v}^{*} v_{y} g(w, z)\right| \leq C\left|v_{y}\right| \leq \frac{C}{s}
$$

because

$$
\left|v_{y}\right|=\left|\partial_{y}\left[h^{*}(u)^{-1} g^{0}(u)^{\prime} u_{y}\right]\right| \leq C\left(\left|u_{y y}\right|+\left|u_{y}\right|^{2}\right) \leq \frac{C}{s} .
$$

Hence,

$$
I_{3} \leq-\eta_{u u}^{*} u_{y} f(w, z)+\frac{C}{s}
$$

Without loss of generality, we can assume $w_{r}>w_{l}$ and therefore, from the monotonicity of $U(\xi)$ established in Proposition 1, it follows that $u_{y} \geq 0$. Moreover, the convexity of $\eta^{*}$ implies $\eta_{u u}^{*}(u, v) \geq 0$. Hence, with the notation

$$
M=\sup \left\{|f(w, z)| \text { for } w \in\left[-\|w\|_{\infty},\|w\|_{\infty}\right] ; z \in\left[-\|z\|_{\infty},\|z\|_{\infty}\right]\right\},
$$

we get

$$
-\eta_{u u}^{*}(u, v) u_{y}(f(w, z)+M) \leq 0 .
$$


Thus, by using the above inequalities, one has

$$
\begin{aligned}
I_{3} & \leq \frac{C}{s}+M \partial_{y}\left[\eta_{u}^{*}(u, v)\right]-M \eta_{u v}^{*}(u, v) v_{y} \\
& \leq M \partial_{y}\left[\eta_{u}^{*}(u, v)\right]+\frac{C}{s} .
\end{aligned}
$$

Finally,

$$
\partial_{s} H+\partial_{y} \widetilde{Q} \leq \frac{C}{s}
$$

where $\widetilde{Q}=Q-M \eta_{u}^{*}(u, v)$. Let $\Phi(y, s)=\frac{1}{\sqrt{s}} \varphi\left(\frac{y}{\sqrt{s}}\right)$, where $\varphi(\xi) \geq 0$ is a test function. Proceeding as before,

$$
\begin{aligned}
\partial_{s}(\Phi H)+\partial_{y}(\Phi \widetilde{Q}) & \leq \frac{C}{S} \Phi+\Phi_{s} H+\Phi_{y} \widetilde{Q} \\
& \leq \frac{C}{s} \Phi+\frac{C}{s} \varphi^{\prime}|\widetilde{Q}|+|H|\left[\frac{1}{s} \varphi+\frac{1}{s^{\frac{3}{2}}} \varphi\right] \\
& \leq \frac{C}{s} B\left(\frac{y}{\sqrt{s}}\right), \text { for any } s>1,
\end{aligned}
$$

where $B$ is a continuous function with compact support. Integrating (21) in $y$, we get

$$
\frac{d}{d s} \int_{-\infty}^{+\infty} \Phi\left(\frac{y}{\sqrt{s}}\right) H(y, s) d y \leq \frac{C}{s}, \text { for any } s>1,
$$

which is exactly $(20)$, since

$$
\begin{aligned}
\int_{-\infty}^{+\infty} \Phi\left(\frac{y}{\sqrt{s}}\right) H(y, s) d y & =\int_{-\infty}^{+\infty} \frac{1}{\sqrt{s}} \varphi\left(\frac{y}{\sqrt{s}}\right) H(y, s) d y \\
& =\int_{-\infty}^{+\infty} \varphi(\xi) H(\xi \sqrt{s}, s) d \xi
\end{aligned}
$$

REMARK 4. In the proof of the energy estimate (20), we do not use the strict hyperbolicity of the relaxing system (conciition (S.2)). This condition is used only in the proof of the convergence established in Theorem 1. Therefore, the pointwise convergence (18) of Theorem 2 holds also in the case of non-strictly hyperbolic systems, if one already knows the strong convergence of its solutions in $L_{l o c}^{p}$, for $p$ sufficiently large. Besides, a necessary condition in the proof of $(20)$ is the parabolicity of the limit equation, namely the decay estimates given in Proposition 1 for the asymptotic states.

\section{REFERENCES}

[1] R. J. DiPerna. Convergence of approximate solutions to conservation laws. Arch. Rational Mech. Anal., 82:27-70, 1983.

[2] L. Hsiao and T.-P. Liu. Convergence to nonlinear diffusion waves for solutions of a system of hyperbolic conservation laws with damping. Comm. Math. Physics, 143:599 605, 1992.

[3] L. Hsiao and T. Luo. Nonlinear diffusive phenomena of entropy weak solutions for a system of quasilinear hyperbolic conservation laws with damping. Quart. Appl. Math., 56:173-189, 1998.

[4] F. Huang and R. Pan. Convergence rate for compressible Euler equations with damping and vacuum. Arch. Rational Mech. Anal., 166:359-376, 2003. 
[5] C. Lattanzio. On the 3-D bipolar isentropic Euler-Poisson model for semiconductors and the driftdiffusion limit. Math. Models Methods Appl. Sci., 10:351-360, 2000.

[6] C. Lattanzio and P. Marcati. The relaxation to the drift-diffusion system for the 3- $D$ isentropic Euler-Poisson model for semiconductors. Discrete Contin. Dynam. Systems, 5(2):449-455, 1999.

[7] P. L. Lions and G. Toscani. Diffusive limit for finite velocity Boltzmann kinetic models. Rev. Mat. Iberoamericana, 13:473-513, 1997.

[8] P. Marcati and A. Milani. The one-dimensional Darcy's law as the limit of a compressible Euler flow. J. Differential Equations, 84:129-147, 1990.

[9] P. Marcati, A. Milani, and P. Secchi. Singular convergence of weak solutions for a quasilinear nonhomogeneous hyperbolic system. Manuscripta Math., 60:49-69, 1988.

[10] P. Marcati and R. Natalini. Weak solutions to a hydrodynamic model for semiconductors and relaxation to the drift-diffusion equation. Arch. Rational Mech. Anal., 129:129-145, 1995.

[11] P. Marcati and B. Rubino. Hyperbolic to parabolic relaxation theory for quasilinear first order systems. J. Differential Equations, 162:359-399, 2000.

[12] F. Murat. Compacité par compensation. Ann. Scuola Norm. Sup. Pisa Cl. Sci. (4), 5:489-507, 1978.

[13] B. Rubino. Porous media flow as the limit of a nonstrictly hyperbolic system of conservation laws. Comm. Partial Differential Equations, 21:1-21, 1996.

[14] B. Rubino. Weak solutions to quasilinear wave equations of Klein-Gordon or sine-Gordon type and relaxation to the reaction-diffusion equations. NoDEA Nonlinear Differential Equations Appl., 4:439-457, 1997.

[15] D. Serre and L. Xsiao. Asymptotic behavior of large weak entropy solutions of the damped $p$-system. J. Partial Diff. Eqs., 10:355-368, 1997.

[16] L. Tartar. Compensated compactness and applications to partial differential equations, volume IV of Nonlinear Analysis and Mechanics: Heriot-Watt Symposium, pages 136-212. Res. Notes in Math. 39, Pitman, 1979. 